\title{
OPEN SARS-CoV-2 virus transfers to skin through contact with contaminated solids
}

\author{
Saeed Behzadinasab $\oplus^{1,4}$, Alex W. H. Chin $\mathbb{1}^{2,4}$, Mohsen Hosseini ${ }^{1}{ }^{1}$, Leo L. M. Poon $\mathbb{1}^{2,3 凶} \&$

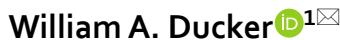

Transfer of SARS-CoV-2 from solids to fingers is one step in infection via contaminated solids, and the possibility of infection from this route has driven calls for increased frequency of handwashing during the COVID-19 pandemic. To analyze this route of infection, we measured the percentage of SARSCoV-2 that was transferred from a solid to an artificial finger. A droplet of SARS-CoV-2 suspension (1 $\mu \mathrm{L}$ ) was placed on a solid, and then artificial skin was briefly pressed against the solid with a light force $(3 \mathrm{~N})$. Transfer from a variety of solids was detected, and transfer from the non-porous solids, glass, stainless steel, and Teflon, was substantial when the droplet was still wet. The viral titer for the finger was $13-16 \%$ or $0.8-0.9$ log less than for the input droplet. Transfer still occurred after the droplet evaporated, but was smaller, 3-9\%. We found a lower level of transfer from porous solids but did not find a significant effect of solid wettability for non-porous solids.

In the period January 2020 to May 2021, about 170 million people contracted COVID-19, and about 3.6 million have died as a result of the illness ${ }^{1}$. The disease is caused by a virus, SARS-CoV-2 (severe acute respiratory syndrome coronavirus 2). In 2020, the US CDC stated that the primary mechanism for transmission is close contacts or inhalation of respiratory droplets ${ }^{2}$. Some diseases can also be transferred via contaminated solids (fomites), and the World Health Organization states that "fomite transmission is considered a likely mode of transmission for SARS-CoV-2"3. A study on hamsters showed that direct inhalation is the main mechanism, but infection via fomite transmission also occurred ${ }^{4}$. Modelling of the pandemic and disease transmission found that up to $25 \%$ of the disease transmissions during lockdown was via fomites ${ }^{5}$. Studies have shown that SARS-CoV-2 remains infective up to 7 days after a droplet is placed on some solids ${ }^{6,7}$, indicating the window of possible infection from solids may be large. This has led to widespread fear of touching communal objects and to health authorities suggesting that people increase the frequency and quality of their hand-washing ${ }^{8}$. A parallel approach is to design coatings for solids that inactivate the virus and to apply these coatings to communal objects ${ }^{9-14}$.

An important question remains unanswered: does the virus actually transfer from contaminated objects to a person's hand? The transfer of virus from the contaminated solid surface to the body is necessary for infection via fomites. In this article, we describe measurements of the percentage transfer of SARS-CoV-2 to skin from a variety of solids.

Following biosafety protocols, we were not able to examine transfer to the skin of living humans. We instead used VITRO-SKIN, an artificial skin, which has many characteristics that are similar to human skin. VITROSKIN has similar wettability ${ }^{15}$, mechanical properties ${ }^{16}$, textural features, and chemistry to human skin (see Supplementary Information.) Others have found VITRO-SKIN to be a good model for human skin ${ }^{16,17}$. An additional advantage of using an artificial skin is that human skin varies from place to place on the body, varies between individuals, and depends on the state of the individual. Use of a constant skin sample enables better resolution of the variations in transfer from different inanimate objects.

We made an artificial finger from polydimethylsiloxane (PDMS) with a modulus similar to human skin and attached the VITRO-SKIN to the PDMS. We contaminated each solid with $1 \mu \mathrm{L}$ of SARS-CoV-2 in suspension in buffer. $1 \mu \mathrm{L}$ is the upper end of the size range of respiratory droplets ${ }^{18}$, much smaller droplets did not allow sufficient resolution of the viral titer.

\footnotetext{
${ }^{1}$ Department of Chemical Engineering and Center for Soft Matter and Biological Physics, Virginia Tech, Blacksburg, VA 24061, USA. ${ }^{2}$ School of Public Health, LKS Faculty of Medicine, The University of Hong Kong, Hong Kong Special Administrative Region, Hong Kong, China. ${ }^{3} \mathrm{HKU}$-Pasteur Research Pole, LKS Faculty of Medicine, The University of Hong Kong, Hong Kong, China. ${ }^{4}$ These authors contributed equally: Saeed Behzadinasab and Alex W. H. Chin. ${ }^{\boxplus}$ email: Ilmpoon@hku.hk; wducker@vt.edu
} 
As described below, our experiments do show that SARS-CoV-2 transfers from solids to skin, even when contact is made after the droplet has evaporated.

\section{Materials and methods}

Details of the materials and their characterization are in Supplementary Information S.1.1-S.1.2.2, S11-S122.

Artificial skin. VITRO-SKIN was purchased from IMS Division of Florida Skincare Testing Inc. (Florida, USA). In this manuscript "artificial skin" refers to VITRO-SKIN. As-received VITRO-SKIN needs to be hydrated to be a good model for human skin. Hydration was achieved in a sterilized, sealed chamber that contained $15 \%$ $\mathrm{w} / \mathrm{w}$ glycerin in water for $16-24 \mathrm{~h}^{16}$. Artificial skin was sterilized with $70 \%$ ethanol prior to the hydration step.

Artificial finger. PDMS was chosen as support for the skin because we were able to make a PDMS support that had about the same deformation as a human finger when subjected to a $3 \mathrm{~N}$ load. Sylgard 184 (base to curing agent $=10: 1$ ) and Sylgard 527 (part A to part $B=1: 1$ ) were prepared separately and the two were thoroughly mixed with a ratio of Sylgard 527:184 $=5: 1$. Subsequently the mixture was poured into hemicylindrical-shaped molds (plastic centrifuge tube, diameter $=15 \mathrm{~mm}$ ) and heated at $80^{\circ} \mathrm{C}$ for $9 \mathrm{~h}$ to cure. The resulting PDMS had a hemicylindrical shape, and the curved side faced the test solid during the transfer (See Supplementary Fig. S5). The in vivo elastic modulus of human skin is $0.04-0.22 \mathrm{MPa}^{19,20}$, which spans the range of $0.13 \mathrm{MPa}$ measured for Sylgard 527:184 $=5: 1^{21}$. The sterilized and hydrated artificial skin was attached to the PDMS hemicylinder using double-sided tape to create the artificial finger.

Transfer experiment. A $1 \mu \mathrm{L}$ droplet of SARS-CoV-2 suspension at $\log _{10} 7.8 \mathrm{TCID} \mathrm{T}_{50} / \mathrm{ml}$ was placed on each test solid (glass, etc.) at $22-23^{\circ} \mathrm{C}$ and relative humidity of $60-70 \%$, and then the artificial skin was contacted to the test solid after waiting for either $10 \mathrm{~s}$ or $30 \mathrm{~min}$. After $10 \mathrm{~s}$, the droplet evaporation was negligible, and so we describe the test solid as "wet"; after $30 \mathrm{~min}$, the droplet had evaporated so the test solid was dry, which we refer to as "evaporated" or "dry". Artificial skin was attached to the curved side of the PDMS immediately before the touch experiment. Contact between artificial skin and the test solid was for $5 \mathrm{~s}$ with a mass of $299.2 \pm 1.2 \mathrm{~g}$, i.e., with a force of $\approx 3 \mathrm{~N}$ (See Supplementary Information S3, Fig. S5). This contact force was chosen after measuring the force that we used to press a checkout credit-card machine button. The $5 \mathrm{~s}$ contact time was longer than what we use to press a credit card reader button, but was chosen to achieve a smaller percentage variation in contact time. The contact area was roughly $1.2 \mathrm{~cm} \times 0.3 \mathrm{~cm}$, resulting in an average pressure of about $1 \mathrm{~atm}$. The flat-cylinder contact was chosen to achieve a well-defined contact. A flat-flat contact would depend critically on alignment; a sphere-flat contact was not possible with the flat sheets of artificial skin that we utilized. An additional critical aspect was that that we needed to ensure that, during contact, the droplet did not run off the edges of the test solid; that would not allow accurate or reproducible accounting of the transfer. The use of a curved skin substrate provided more space within the liquid meniscus for the droplet to remain between the solids during contact. This was confirmed using dye tests prior to the experiments (Supplementary Fig. S5C).

SARS-CoV-2 assay. After the finger was separated from the test solid, the artificial skin was removed from the PDMS and the skin was soaked in $200 \mu \mathrm{L}$ of viral transport medium (Earle's balanced salt solution, which was supplemented with $0.5 \%(\mathrm{w} / \mathrm{v})$ bovine serum albumin and $0.1 \%(\mathrm{w} / \mathrm{v})$ glucose, $\mathrm{pH}=7.4)$ at room temperature for $30 \mathrm{~min}$ to elute the SARS-CoV-2 virus (from the Hong Kong index case). Subsequently, the eluted viral suspension was assayed by $50 \%$ tissue culture infective dose $\left(\mathrm{TCID}_{50}\right.$ ) assay in Vero E6 cells to determine how effective the eluent was in infecting mammalian cells ${ }^{22,23}$. Supplementary Information, Section S1.2, contains further information on the SARS-CoV-2 culture and RT-qPCR.

Statistical analysis. We and others find that the errors in $\log \left(\mathrm{TCID}_{50}\right)$ are distributed normally, and therefore all statistics were obtained after a log transformation of the raw $\mathrm{TCID}_{50}$ data. Data for Fig. 2, Supplementary Fig. S8 and S9 was analyzed using N-way ANOVA in MATLAB R2021a (https://www.mathworks.com/produ cts/matlab.html) with two factors, material (glass, stainless steel, etc.) and wet/evaporated. The population marginal means were estimated using Post Hoc multiple comparison (Dunn-Sidák’s approach). Additionally, we did a regression analysis with three factors - porosity (categorical), wettability (numerical from contact angle) and wet/evaporated. $\mathrm{p}<0.001$ for coefficients for porosity and wet/dry. Unless otherwise stated, errors are $95 \%$ confidence intervals.

\section{Results}

When a droplet of SARS-CoV-2 suspension is placed on a solid, the virus can be transferred to a finger, even when the finger has only a brief $(5 \mathrm{~s})$ and light $(3 \mathrm{~N})$ touch to the solid that one may do when pressing a button. Figure 1 shows the transfer ratio, $T$, which is the ratio of the infectivity on the finger compared to the infectivity of the original droplet:

$$
T=\frac{N_{\text {finger }}}{N_{\text {initial }}} \times \frac{1}{R} \times 100 \%
$$

where $N_{\text {finger }}$ is the infectivity measured from liquid extracted from the finger after contact with the solid, as measured by TCID $_{50}$ assay in Vero E6 (African green monkey) cells, and $N_{\text {initial }}$ is the infectivity measured in the droplet that was initially placed on the test solid. $R$ is the recovery rate for extracting virus from the finger. More detail is provided in Supplementary Information, Section S4. 


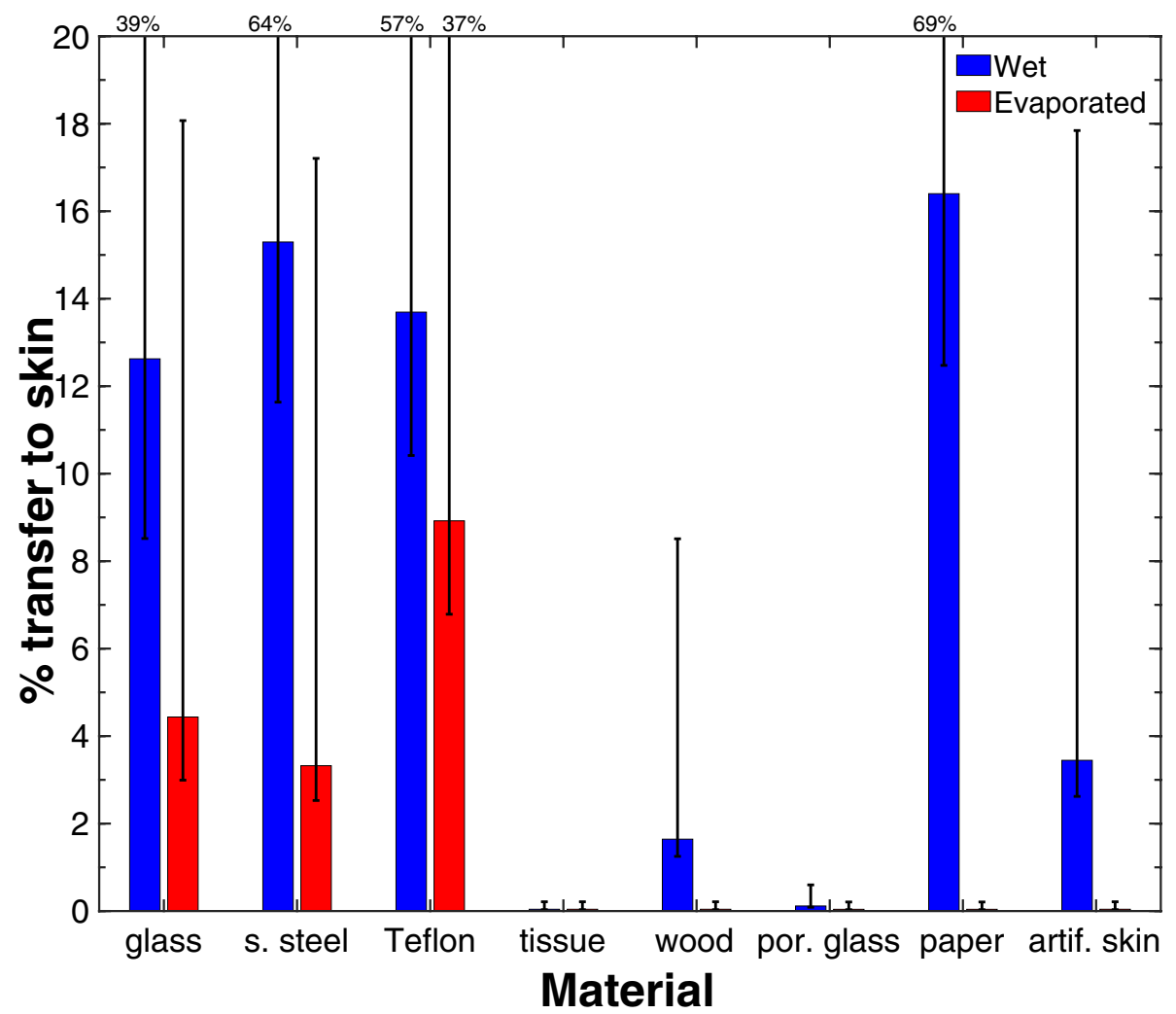

Figure 1. Mean percentage transfer to an artificial finger from various solid material during a $5 \mathrm{~s}$ contact with a $3 \mathrm{~N}(299.2 \pm 1.2 \mathrm{~g})$ load with no lateral motion (i.e., no rubbing). Error bars are the comparison intervals from ANOVA with two factors: the material and the time, which corresponds to wet or evaporated. MATLAB was used to create the figure and to calculate the ANOVA. The error bar is asymmetric because of the exponential distribution of measurement residuals. When the upper bound is off-scale, it is indicated by a number. The time to adsorb the droplet on porous surfaces was as follows: tissue and porous glass, immediate; wood, 1-2 min; and paper, $2.5 \mathrm{~min}$. Note that the transfer is high for non-porous solids and is measurable when the $1 \mu \mathrm{L}$ test drop is wet $(10 \mathrm{~s})$ or has evaporated $(30 \mathrm{~min})$. Each data point was obtained from three independent measurements that are tabulated in Supplementary Table S1.

We examined transfer for two conditions: (1) when it was freshly placed on the solid (after $10 \mathrm{~s}$ ) and (2) after it had evaporated $(30 \mathrm{~min})$. The transfer percentage for wet droplets on the three common non-porous solids - glass, stainless steel and Teflon-are in the range 13-16\% (0.8-0.9 log less than the input) (Fig. 1). These numbers are quite high: the amount of virus transferred to the skin after touching the solid is about $15 \%$ as great as if the droplet landed directly on the finger. When the droplet was allowed to dry, about 3-9\% (1-1.5 $\log$ less than the input) was transferred. This evokes the scenario when an infected individual deposits a droplet and half an hour later a second individual who touches the dry surface could still transfer 3-9\% of the original virus onto their fingers. This is not a large reduction by microbiological standards; for comparison, sanitizers and disinfectants are considered effective when they leave less than $0.1 \%$ of the germ, i.e., at least a $30-150$ times greater reduction. The results reinforce the idea that hand-washing is important before touching one's face. Note that our method of calculating the transfer includes any inactivation of the virus that occurs on the test solid prior to contact with the finger. In some cases, this could be substantial. For example, in our prior work, we showed that only $31 \%$ of the virus was recovered from glass after $30 \mathrm{~min}$, even with no skin contact ${ }^{10}$. We think that the fraction of the initial dose that ends up on the finger is the relevant quantity. We note that for the transfer percentage of the dried droplets may be slightly greater than indicated, as described in Supplementary Information. A separate experiment with $1 / 5$ th of the viral loading on glass revealed that, in this range, the vial load did not affect the transfer.

For the remaining results, we directly compare $\mathrm{TCID}_{50} / \mathrm{mL}$ values, which are shown in Fig. 2. Figure 2, Supplementary Figs. S8 and S9 also show population marginal means. There is a significant decrease in viral transfer after the droplet evaporates $(p<0.0001)$, which is more clearly shown in Supplementary Fig. S8. This is as expected; once the liquid evaporates, there is no liquid meniscus to transport the virus to the finger.

We originally hypothesized that a more hydrophobic solid, i.e., a solid with a greater water receding angle, would transfer more virus. This was based on the idea that a greater volume of liquid would transfer from a hydrophobic solid, and therefore, more virus could be transferred in that greater volume. We examined this hypothesis by including Teflon as one of the samples. Teflon has an unusually high receding angle $\left(96^{\circ} \pm 9^{\circ}\right)$ whereas clean glass has a low contact angle, $11^{\circ} \pm 7^{\circ}$ (Table 1 ). There was no significant difference between the 


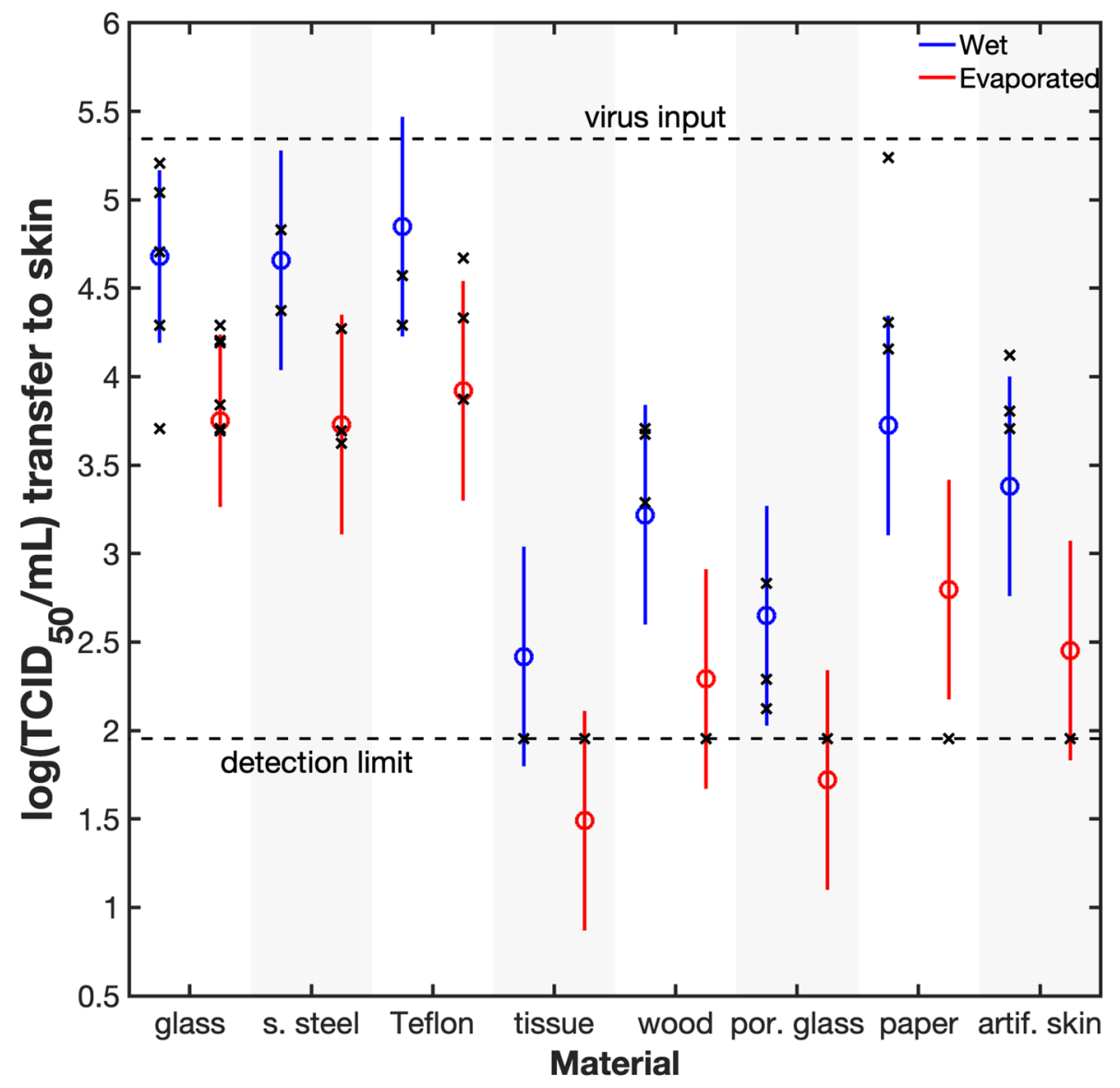

Figure 2. The $\log \left(\mathrm{TCID}_{50} / \mathrm{mL}\right)$ values $(\mathrm{x})$ and population marginal means (o) on artificial skin after transfer from various solids. The population marginal means were calculated from Post Hoc comparison using N-way ANOVA (two factors: material and wet/evaporated) in MATLAB using the Dunn-Sidák's approach. The bars represent the comparison interval; two groups are statistically different if their intervals do not overlap. Transfer is detectable for all solids, except tissue, wood (dry), porous glass (dry), and paper (dry), and these data points have been plotted on the detection limit of 90 . Transfer is greater for wet solids than for dry and there is no significant difference among common non-porous solids. MATLAB was used to create the figure.

\begin{tabular}{|l|l|l|}
\hline \multirow{2}{*}{ Solids } & \multicolumn{2}{|l|}{ Contact angle } \\
\cline { 2 - 3 } & Advancing & Receding \\
\hline Glass & $26 \pm 13$ & $11 \pm 7$ \\
\hline Stainless steel & $84 \pm 15$ & $40 \pm 23$ \\
\hline Teflon & $111 \pm 6$ & $96 \pm 9$ \\
\hline Porous glass & 0 & 0 \\
\hline Paper & $96 \pm 12$ & $11 \pm 3$ \\
\hline Artificial skin & $89 \pm 6$ & $<10$ \\
\hline
\end{tabular}

Table 1. Mean water contact angle measurements of test solids. The figure after \pm sign is the $95 \%$ confidence interval. The contact angle was measured $10 \mathrm{~s}$ after the droplet was placed on the solid.

transfer for the two substances (see Fig. 2 and Supplementary Fig. S9). We then tested the mass of liquid that was transferred (Supplementary Fig. S7), and found that the mass transfer was also similar, which possibly explains why the viral titer did not depend on the contact angle for non-porous solids.

Porosity, however, does play a key role. We examined three porous objects (tissue, wood, and porous glass) that were sufficiently hydrophilic that the droplet could penetrate into the solid. Each of these porous solids had less transfer than each of the three non-porous solids (glass, stainless steel, or Teflon). This is evident for wet and dried solids considered separately (Fig. 1) and for wet and dried samples considered together (Supplementary 
Fig. S9). The most direct comparison is between porous and non-porous glass, where the material is the same yet the transfer is completely different. The explanation is fairly simple. For wet surfaces, the droplet lies mainly within the porosity at the time of contact with the skin, and so less of the droplet is transferred to skin. For dried droplets, we expect that much of the virus is dried into the interior of the porosity and is not available on the surface for dry, solid-to-solid transfer. We did some further tests on the effect of porosity using real time quantitative polymerize chain reaction (RT-qPCR) measurements. These RT-qPCR measurements confirm that the virus does become trapped in interior pores (see Supplementary Fig. S10). We expect that the effect of porosity would be contingent on the interior pores of the solid being wettable. Thus, we would not expect the droplet to penetrate porous Teflon, so we predict transfer would still be high from this material.

Recent work has also tested the transfer of SARS-CoV-2 from cardboard or plastic to pig skin ${ }^{24}$. That work showed that transfer was greater from plastic than from cardboard. The authors state that the plastic was nonporous and that the cardboard was porous, and make a similar conclusion that the lack of transfer for cardboard is due to the drop being absorbed into the porous surface.

The paper material provides an interesting example of a porous material. Transfer from paper was initially (after $10 \mathrm{~s}$ ) very high whereas transfer after the droplet evaporated $(30 \mathrm{~min})$ was very low. This is explained though an understanding of how the wettability of the material affects access to the porosity. If the chemistry of pores is sufficiently hydrophobic, then the water cannot spontaneously enter the pores and the porosity is not accessed. Although paper is porous, there is an induction time for wetting: for the particular paper that we used, the paper goes from non-wetting and impermeable to wetting and permeable after about 2.5 min of contact with a $1 \mu \mathrm{L}$ droplet (See Supplementary Figure S12). Our "wet" experiment was conducted after $10 \mathrm{~s}$, during the impermeable period. Without access to the porosity, the SARS-CoV-2 suspension behaved as if it were on a nonporous solid, the droplet was transferred to the finger and we recorded a high transfer ratio. After $2.5 \mathrm{~min}$., the viral suspension penetrated into the paper, and subsequently dried into the pore space, presumably depositing the virus onto the paper fibers on the interior of the paper. The transfer experiment after 30 min showed a very low level of transfer because those interior surfaces were not accessible to the finger during transfer.

A further interesting aspect of porosity concerns the fate of the SARS-CoV-2 in the porosity. We also measured the TCID ${ }_{50}$ of liquid used to extract virus from the dried solids after contact with the finger (data not shown) using the same procedure as for extraction from the skin (see Supplementary Information S.1.2.4). We were not surprised that the levels were very low for porous solids. For porous glass, tissue, and wood, $\mathrm{TCID}_{50} / \mathrm{mL}$ was below the detection limit. However, for paper, $\log \left[\mathrm{TCID}_{50} / \mathrm{mL}\right]$ was 3.5. This indicates that SARS-CoV-2 can, to some extent, be stored in dried paper, and resuspended in an active state.

Finally, we used linear regression to make a predictive model for the transfer as a function of the wet/dry state, the porosity, and the wettability. This model performed well and is described in Supplementary Fig. S13.

\section{Discussion}

People use a huge range of contact trajectories when touching surfaces, for example, different pressures, different times of contact, and different rubbing actions. In this study we considered only brief and low-pressure contact with no rubbing or translation in contact. It is well-established that rubbing causes friction and increases wear and transfer between solids, and in particular that microbial transfer is increased significantly by rubbing ${ }^{25}$. We therefore expect that transfer of SARS-CoV-2 would also be enhanced by rubbing.

Infected patients can shed droplets with high concentrations of SARS-CoV-2 ${ }^{26,27}$. For example, Pan et al. showed a median of $7.5 \times 10^{5}$ and a high of $10^{7}$ gene copies per $\mathrm{mL}$ in sputum ${ }^{26}$. Our research shows that, on non-porous solids, $13-16 \%$ of this virus can be transferred to skin after being deposited on a solid when the drop is still wet and 3-9\% can be transferred even $30 \mathrm{~min}$ later, when the droplet is dry. This still leaves a large dose of virus on the finger. Clearly one more step is required to transfer the virus to the respiratory system: transfer from the finger to the nose or mouth. The overall transmission from solid to skin to respiratory system depends on the product of the individual transfer rates, and would be lower. The final dose transferred to the respiratory system should be compared to the infectious dose for a human, which unfortunately is not known. Studies of Syrian hamsters indicate that the infective does for these susceptible animals is only 5 infectious particles ${ }^{28}$. Clearly hand washing can reduce both the initial contamination of surfaces and intercept the virus before it is transferred from fingers to the respiratory system.

Infection via fingers depends also on the longevity of the virus on human skin: the virus must survive long enough on skin to be transferred. Harbourt et al. reported the stability of SARS-CoV-2 on dead porcine (pig) $\operatorname{skin}^{29}$. They found the virus remained stable for 4 days at $22 \pm 2{ }^{\circ} \mathrm{C}$, and $8 \mathrm{~h}$ at $37 \pm 2{ }^{\circ} \mathrm{C}$ (relative humidity of $40-50 \%)$. Hirose et al. measured viability of the SARS-CoV-2 virus on a dead human skin model ${ }^{30}$. They showed the virus can survive on the skin model for up to $9 \mathrm{~h}$ at $25^{\circ} \mathrm{C}$ and relative humidity of $45-55 \%$. These longevity studies suggest that once SARS-CoV-2 has been transferred to the skin, there is a long period where there is an opportunity to transfer viable virus to the eye, nose, or mouth.

\section{Conclusions}

In summary, our research demonstrates substantial transfer of SARS-CoV-2 virus from a variety of solids to an artificial finger after a brief, light force and no rubbing. The transfer is lower but still occurs after the droplet has dried. When the droplet penetrates a porous solid, such as wood or tissue, the transfer is low.

\section{Data availability}

The data that supports the findings of this study are available within the Supplementary Information. 
Received: 11 June 2021; Accepted: 6 October 2021

Published online: 24 November 2021

\section{References}

1. World Health Organization. WHO Coronavirus Disease (COVID-19) Dashboard. https://covid19.who.int/ (2021).

2. Center for Disease Control. Cleaning and Disinfection for Households. https://www.cdc.gov/coronavirus/2019-ncov/prevent-getti ng-sick/cleaning-disinfection.html (2020).

3. World Health Organization. Transmission of SARS-CoV-2: Implications for Infection Prevention Precautions. https://www.who.int/ news-room/commentaries/detail/transmission-of-sars-cov-2-implications-for-infection-prevention-precautions (2020).

4. Sia, S. F. et al. Pathogenesis and transmission of SARS-CoV-2 in golden hamsters. Nature 583, 834-838 (2020).

5. Meiksin, A. Dynamics of COVID-19 transmission including indirect transmission mechanisms: A mathematical analysis. Epidemiol. Infect. 148, e257. https://doi.org/10.1017/S0950268820002563 (2020).

6. Van Doremalen, N. et al. Aerosol and surface stability of SARS-CoV-2 as compared with SARS-CoV-1. N. Engl. J. Med. 382, 1564-1567 (2020).

7. Chin, A. W. H. et al. Stability of SARS-CoV-2 in different environmental conditions. Lancet Microbe 1, e10. https://doi.org/10. 1016/S2666-5247(20)30003-3 (2020)

8. Alzyood, M., Jackson, D., Aveyard, H. \& Brooke, J. COVID-19 reinforces the importance of handwashing. J. Clin. Nurs. 29, 2760-2761. https://doi.org/10.1111/jocn.15313 (2020).

9. Behzadinasab, S., Chin, A., Hosseini, M., Poon, L. \& Ducker, W. A. A surface coating that rapidly inactivates SARS-CoV-2. ACS Appl. Mater. Interfaces 12, 34723-34727 (2020).

10. Hosseini, M., Chin, A. W., Behzadinasab, S., Poon, L. L. \& Ducker, W. A. Cupric oxide coating that rapidly reduces infection by SARS-CoV-2 via solids. ACS Appl. Mater. Interfaces 13, 5919-5928 (2021).

11. Hutasoit, N. et al. Sars-CoV-2 (COVID-19) inactivation capability of copper-coated touch surface fabricated by cold-spray technology. Manuf. Lett. 25, 93-97 (2020).

12. Hasan, J. et al. Antiviral nanostructured surfaces reduce the viability of SARS-CoV-2. ACS Biomater. Sci. Eng. 6, 4858-4861 (2020).

13. Hosseini, M., Behzadinasab, S., Benmamoun, Z. \& Ducker, W. A. The viability of SARS-CoV-2 on solid surfaces. Curr Opin Colloid Interface Sci. 55101481. https://doi.org/10.1016/j.cocis.2021.101481 (2021).

14. Hosseini, M., Behzadinasab, S., Chin, A., Poon, L. \& Ducker, W. A. Reduction of infectivity of SARS-CoV-2 by zinc oxide coatings. ACS Biomater. Sci. Eng. (2021).

15. Ginn, M., Noyes, C. \& Jungermann, E. The contact angle of water on viable human skin. J. Colloid Interface Sci. 26, 146-151 (1968).

16. Jermann, R., Toumiat, M. \& Imfeld, D. Development of an in vitro efficacy test for self-tanning formulations. Int. J. Cosmet. Sci. 24, 35-42 (2002).

17. Chen, S. \& Bhushan, B. Nanomechanical and nanotribological characterization of two synthetic skins with and without skin cream treatment using atomic force microscopy. J. Colloid Interface Sci. 398, 247-254 (2013).

18. Duguid, J. The size and the duration of air-carriage of respiratory droplets and droplet-nuclei. Epidemiol. Infect. 44, 471-479 (1946).

19. Pailler-Mattei, C., Bec, S. \& Zahouani, H. In vivo measurements of the elastic mechanical properties of human skin by indentation tests. Med. Eng. Phys. 30, 599-606 (2008).

20. Diridollou, S. et al. In vivo model of the mechanical properties of the human skin under suction. Skin Res. Technol. 6, 214-221 (2000).

21. Palchesko, R. N., Zhang, L., Sun, Y. \& Feinberg, A. W. Development of polydimethylsiloxane substrates with tunable elastic modulus to study cell mechanobiology in muscle and nerve. PLoS ONE 7, e51499 (2012).

22. Chan, K. et al. Analytical sensitivity of rapid influenza antigen detection tests for swine-origin influenza virus (H1N1). J. Clin. Virol. 45, 205-207 (2009).

23. Malenovska, H. Virus quantitation by transmission electron microscopy, TCID50, and the role of timing virus harvesting: A case study of three animal viruses. J. Virol. Methods 191, 136-140 (2013)

24. Johnson, G. T. et al. Evaluation of the survivability of SARS-CoV-2 on cardboard and plastic surfaces and the transferability of virus from surface to skin. Occup. Dis. Environ. Med. 9, 63-73 (2021).

25. Zhao, P. et al. Physical factors that affect microbial transfer during surface touch. Build. Environ. 158, 28-38 (2019).

26. Pan, Y., Zhang, D., Yang, P., Poon, L. L. \& Wang, Q. Viral load of SARS-CoV-2 in clinical samples. Lancet Infect. Dis. 20, 411-412 (2020).

27. Yu, F. et al. Quantitative detection and viral load analysis of SARS-CoV-2 in infected patients. Clin. Infect. Dis. 71, 793-798 (2020).

28. Rosenke, K. et al. Defining the Syrian hamster as a highly susceptible preclinical model for SARS-CoV-2 infection. Emerg. Microbes Infect. 9, 2673-2684 (2020).

29. Harbourt, D. E. et al. Modeling the stability of severe acute respiratory syndrome coronavirus 2 (SARS-CoV-2) on skin, currency, and clothing. PLoS Negl. Trop. Dis. 14, e0008831 (2020).

30. Hirose, R. et al. Survival of severe acute respiratory syndrome coronavirus 2 (SARS-CoV-2) and influenza virus on human skin: Importance of hand hygiene in coronavirus disease 2019 (COVID-19). Clin. Infect. Dis. https://doi.org/10.1093/cid/ciaa1517 (2020).

\section{Acknowledgements}

The authors acknowledge use of Microscopy facilities within the NCFL at Virginia Tech. We thank Professor Thomas Staley for grinding the glass particles to an appropriate size, Kevin Holshouser for fabricating the transfer apparatus, and Matty Ducker for the linear regression in Figure S13. This work was supported by the National Science Foundation under Grant No. CBET-1902364, the Health and Medical Research Fund (COVID190116), and the National Institute of Allergy and Infectious Diseases (contract HHSN272201400006C).

\section{Author contributions}

W.A.D. and L.L.M.P. conceived of the experiments and W.A.D. designed the transfer apparatus. W.A.D. and S.B. designed the artificial finger and designed the experiments. S.B. prepared and characterized the samples unless otherwise noted. M.H. fabricated the porous glass samples. Analysis of data was done by W.A.D., S.B., A.W.H.C., M.H., and L.L.M.P. The preparation of SARS-CoV-2 and experiments containing the virus were done by A.W.H.C. Supervision was done by W.A.D. and L.L.M.P. The manuscript was written by S.B. and W.A.D. with input from all authors. 


\section{Competing interests}

WAD declares part ownership in a startup company to produce surface coatings. No other authors declare competing interests.

\section{Additional information}

Supplementary Information The online version contains supplementary material available at https://doi.org/ 10.1038/s41598-021-00843-0.

Correspondence and requests for materials should be addressed to L.L.M.P. or W.A.D.

Reprints and permissions information is available at www.nature.com/reprints.

Publisher's note Springer Nature remains neutral with regard to jurisdictional claims in published maps and institutional affiliations.

(c) (i) Open Access This article is licensed under a Creative Commons Attribution 4.0 International License, which permits use, sharing, adaptation, distribution and reproduction in any medium or format, as long as you give appropriate credit to the original author(s) and the source, provide a link to the Creative Commons licence, and indicate if changes were made. The images or other third party material in this article are included in the article's Creative Commons licence, unless indicated otherwise in a credit line to the material. If material is not included in the article's Creative Commons licence and your intended use is not permitted by statutory regulation or exceeds the permitted use, you will need to obtain permission directly from the copyright holder. To view a copy of this licence, visit http://creativecommons.org/licenses/by/4.0/.

(C) The Author(s) 2021 